\title{
SURVIVAL OF INHOMOGENEOUS GALTON-WATSON PROCESSES
}

\author{
ERIK BROMAN, ${ }^{*}$ Chalmers University of Technology \\ RONALD MEESTER, ${ }^{* *}$ VU University Amsterdam
}

\begin{abstract}
We study the survival properties of inhomogeneous Galton-Watson processes. We determine the so-called branching number (which is the reciprocal of the critical value for percolation) for these random trees (conditioned on being infinite), which turns out to be an almost sure constant. We also shed some light on the way in which the survival probability varies between the generations. When we perform independent percolation on the family tree of an inhomogeneous Galton-Watson process, the result is essentially a family of inhomogeneous Galton-Watson processes, parameterized by the retention probability $p$. We provide growth rates, uniformly in $p$, of the percolation clusters, and also show uniform convergence of the survival probability from the $n$th level along subsequences. These results also establish, as a corollary, the supercritical continuity of the percolation function. Some of our results are generalizations of results in Lyons (1992).
\end{abstract}

Keywords: Inhomogeneous Galton-Watson tree; continuity of percolation functions; branching number

2000 Mathematics Subject Classification: Primary 60K37; 60J80; 60K35

\section{Introduction and main results}

We start by defining the main object of study in this paper, namely the inhomogeneous Galton-Watson processes. Start with a root $o$, and let $L_{1}$ be the distribution of the (random) number of offspring of the root. Proceed by letting each child (if any) of the root have an independent and identically distributed (i.i.d.) number of offspring with distribution $L_{2}$, and also let these offspring be independent of the number of children of the root. Given a sequence $\left\{L_{n}\right\}_{n=1}^{\infty}$, we let $L_{n}$ be the offspring distribution of every individual of generation $n-1$. Sometimes we will treat $L_{n}$ as a random variable rather than as a distribution, this is a standard abuse of notation. The root is considered to be generation 0 . Observe that, if the distributions $\left\{L_{n}\right\}_{n=1}^{\infty}$ are all the same, we obtain a regular Galton-Watson process. Also, observe that, if $\mathrm{P}\left(L_{n}=l_{n}\right)=1$ for every $n$ and some sequence of numbers $\left\{l_{n}\right\}_{n=1}^{\infty}$, we almost surely obtain a (deterministic) spherically symmetric tree, that is, a rooted tree in which any two vertices in the same generation have the same degree.

We denote the random family tree of such an inhomogeneous Galton-Watson process by $T$. We will let $\bar{T}$ be a tree with distribution equal to $T$ conditioned on survival, and we will also let $I \subset \bar{T}$ be the tree that consists of those vertices $x \in \bar{T}$ (and the edges between them) that

Received 28 February 2008; revision received 5 June 2008.

* Postal address: Mathematical Sciences, Chalmers University of Technology, SE-412 96 Göteborg, Sweden.

Email address: broman@math.chalmers.se

** Postal address: Department of Mathematics, VU University Amsterdam, De Boelelaan 1081a, 1081 HV Amsterdam,

The Netherlands. Email address: rmeester@few.vu.nl 
have infinitely many descendants in $\bar{T}$. We will denote by $T_{n}, \bar{T}_{n}$, and $I_{n}$ the number of points in the $n$th generation of $T, \bar{T}$, and $I$, respectively.

It is well known (see, e.g. [5]) and not hard to see that $I$ is itself the family tree of an inhomogeneous Galton-Watson process; we will use this fact later on.

For inhomogeneous Galton-Watson processes, we define the survival probability $\theta_{n}$ from the $n$th generation, that is,

$$
\theta_{n}:=\lim _{m \rightarrow \infty} \mathrm{P}\left(T_{m}>0 \mid T_{n}=1\right) .
$$

For an infinite tree, a cutset $\pi$ is defined to be a finite set of edges such that every infinite path starting at the origin must contain at least one edge of the cutset. We denote by $\Pi$ the set of all such cutsets. Any infinite tree $S$ has a so-called branching number which is defined as follows.

Definition 1.1. The branching number of an infinite tree $S$ with $\operatorname{root} o$ is denoted by br $S$ and defined by

$$
\operatorname{br} S:=\sup \left\{\lambda ; \inf _{\pi \in \Pi} \sum_{e \in \pi} \lambda^{-|e|}>0\right\} .
$$

The branching number is a very important property for trees (see [6]). For instance, it is known (see [4]) that the critical density $p_{\mathrm{c}}(S)$ for independent percolation (we are assuming that the reader is familiar with the concept of percolation, otherwise please see [3] for a general overview) on $S$ is the reciprocal of the branching number, that is,

$$
p_{\mathrm{c}}(S)=\frac{1}{\mathrm{br} S} .
$$

Closely related to the branching number is the lower growth number $\operatorname{gr} S$, which is defined by

$$
\underline{\operatorname{gr} S}:=\liminf _{n \rightarrow \infty} S_{n}^{1 / n}
$$

where $S_{n}$ denotes the number of vertices in the $n$th generation of $S$. It is not hard to see that we always have $\operatorname{br} S \leq \operatorname{gr} S$, while equality is not always true. It is however well known that if $S$ is spherically symmetric then $\operatorname{br} S=\operatorname{gr} S$.

We start with the following simple survival criterion. This result is essentially contained in Proposition 4.15 of [5], but we give a different proof based on even earlier work in [1]. The reason is that some of the elements in the proof will be used again later in this paper.

Proposition 1.1. For any inhomogeneous Galton-Watson process with offspring distributions $\left\{L_{n}\right\}_{n=1}^{\infty}$, we have

$$
\liminf _{n \rightarrow \infty}\left(\mathrm{E}\left[T_{n}\right]\right)^{1 / n}<1 \Longrightarrow \lim _{n \rightarrow \infty} \mathrm{P}\left(T_{n}>0\right)=0 .
$$

Furthermore, if

$$
\sup _{n} \mathrm{E}\left[L_{n}^{2}\right]=C_{1}<\infty
$$

and

$$
\inf _{n} \mathrm{E}\left[L_{n}\right]=C_{2}>0
$$

then we also have

$$
\liminf _{n \rightarrow \infty}\left(\mathrm{E}\left[T_{n}\right]\right)^{1 / n}>1 \Longrightarrow \lim _{n \rightarrow \infty} \mathrm{P}\left(T_{n}>0\right)>0 .
$$


Next we have a result concerning the branching number of $\bar{T}$. A priori this is a random variable, but it turns out that br $\bar{T}$ is an almost sure constant (under mild conditions).

Theorem 1.1. Consider an inhomogeneous Galton-Watson process with offspring distributions $\left\{L_{n}\right\}_{n=1}^{\infty}$ satisfying (1.1) and (1.2). Also, assume that

$$
\liminf _{n \rightarrow \infty} \mathrm{E}\left[T_{n}\right]^{1 / n}>1 .
$$

Then we have $\operatorname{br} \bar{T}=\liminf _{n \rightarrow \infty} \mathrm{E}\left[T_{n}\right]^{1 / n},[\bar{T}]$-almost surely.

We make some remarks about this result.

Remarks. 1. In [5] it was proved that, almost surely,

$$
\operatorname{br} \bar{T}=\liminf _{n \rightarrow \infty} \mathrm{E}\left[T_{n}\right]^{1 / n},
$$

under the assumption that $\sup _{n}\left\|L_{n}\right\|_{\infty}<\infty$. It was claimed in [5] that this assumption cannot be weakened much; our results show that if we add the very natural condition (1.3) then in fact we can significantly weaken the assumptions.

2. Naively, we might believe that this result would follow from easy arguments. For instance, we might try the following approach. Define a new inhomogeneous Galton-Watson tree $T^{\prime}$ by performing percolation on $T$, with the probability of $T^{\prime}$ being open equal to $p$. Depending on whether $p$ is smaller or greater than $1 / \lim \inf \mathrm{E}\left[T_{n}\right]^{1 / n}$, we find from Proposition 1.1 that $T^{\prime}$ dies out almost surely or, respectively, survives with positive probability, concluding the argument. However, we then miss the point that the fact that $T^{\prime}$ survives with positive probability if $p>1 / \lim \inf \mathrm{E}\left[T_{n}\right]^{1 / n}$ does not lead to the conclusion that brT $\geq \lim \inf \mathrm{E}\left[T_{n}\right]^{1 / n}$. Indeed, it is imaginable that, with positive probability, brT $=\lim \inf \mathrm{E}\left[T_{n}\right]^{1 / n}-\delta$ for some positive $\delta$ and that, with positive probability, brT $=\lim \inf \mathrm{E}\left[T_{n}\right]^{1 / n}$. If this were true, $T$, would still survive with positive probability for the indicated $p$.

The following result about the behavior of $\theta_{n}$ will be needed in the proof of Theorem 1.1, but it is also quite interesting in its own right. It is not to be expected that $\theta_{n}$ is in general bounded away from 0 , since we can always insert any finite number of generations of degree 1 in the tree. However, it is the case that there is a subsequence along which $\theta_{n}$ is bounded away from 0 .

Proposition 1.2. Consider an inhomogeneous Galton-Watson process with offspring distributions $\left\{L_{n}\right\}_{n=1}^{\infty}$ satisfying (1.1), (1.2), and (1.3). Then there exists a sequence $\left\{n_{k}\right\}_{k=1}^{\infty}$ of increasing integers and a constant $C>0$ such that, for all $k \geq 1$,

$$
\theta_{n_{k}} \geq C
$$

Next, we study the bond percolation on $I$. Note that $p_{\mathrm{c}}(I)=p_{\mathrm{c}}(\bar{T})$, since pruning a tree does not change its critical probability. We already noted that $I$ itself is the family tree of an inhomogeneous Galton-Watson process, and when we perform independent bond percolation on $I$, the resulting component of the origin, to be denoted by $I^{p}$, also constitutes a family tree of an inhomogeneous Galton-Watson process. Therefore, general results about inhomogeneous Galton-Watson processes automatically apply to percolation on $I$. However, being equipped with a parameter $p$ now, we will derive survival estimates uniformly in $p$. We remark that a special case of inhomogeneous Galton-Watson processes results from starting with a deterministic spherically symmetric tree and performing percolation on that tree. One 
more piece of notation: the number of vertices in $I^{p}$ at distance $n$ from the root is denoted by $I_{n}^{p}$. Also, in this paper we use various coupling constructions. To facilitate this, all processes, for all values of $p$, are jointly constructed in the obvious way. Consequently, as in the previous example, we will express the $p$-dependence in the events rather than in the measure.

In light of Theorem 1.1, we might expect that, for any $\varepsilon>0$,

$$
\lim _{n \rightarrow \infty} \mathrm{P}\left(0<I_{n}^{p}<\left((1-\varepsilon) \operatorname{br} \bar{I}^{p}\right)^{n}\right)=0 .
$$

In fact, we have the next, much stronger statement.

Theorem 1.2. Consider an inhomogeneous Galton-Watson process satisfying (1.1), (1.2), and (1.3), with family tree $T$, and let $\varepsilon>0$. For $p_{\mathrm{c}}(\bar{T})<p_{1} \leq 1$, it is the case that

$$
\lim _{n \rightarrow \infty} \mathrm{P}\left(0<I_{n}^{p}<\left((1-\varepsilon) \operatorname{br} \bar{p}_{1}\right)^{n}\right)=0
$$

uniformly in $p \in\left[p_{1}, 1\right]$.

Note that the pointwise (in $p$ ) convergence in Theorem 1.2 is almost a triviality. The whole point of the theorem is proving the uniform convergence.

Theorem 1.2 combined with Proposition 1.2 will in turn lead us to our next result. Here we define

$$
\theta(p):=\mathrm{P}\left(\left|I^{p}\right|=\infty\right) .
$$

Proposition 1.3. Consider an inhomogeneous Galton-Watson process satisfying (1.1), (1.2), and (1.3), and let $p_{1}>p_{\mathrm{c}}(\bar{T})$. Then there exists a sequence of increasing integers $\left\{n_{k}\right\}_{k=1}^{\infty}$ such that

$$
\theta(p)=\lim _{k \rightarrow \infty} \mathrm{P}\left(I_{n_{k}}^{p}>0\right)
$$

uniformly on $\left[p_{1}, 1\right]$.

This result also leads to continuity of the percolation function above $p_{\mathrm{c}}$ for random trees.

Corollary 1.1. Consider an inhomogeneous Galton-Watson process satisfying (1.1), (1.2), and (1.3). Then the percolation function $\theta(p)$ is continuous above $p_{\mathrm{c}}(\bar{T})$. In particular, on any spherically symmetric tree $S$ with uniformly bounded degrees, the percolation function is continuous above $p_{\mathrm{c}}(S)$.

In fact, we can also use Theorem 1.1 to construct a more or less classical proof of this result. As an interesting side remark, we mention that the route via Proposition 1.3 also has a counterpart on $\mathbb{Z}^{d}$ and gives a new proof for the continuity of the percolation function in that context. This proof does in fact give a rate of convergence for the natural approximations of the percolation function; we discuss these continuity matters in Section 4.

In contrast to our last corollary we have the following example of a tree for which the percolation function is not continuous above $p_{\mathrm{c}}$. To construct such a tree, we use a result in [5], a special case of which says that there is percolation with positive probability on a spherically symmetric tree $S$ with parameter $p$ if and only if

$$
\sum_{n=1}^{\infty} \frac{p^{-n}}{S_{n}}<\infty .
$$

To construct an example, we first take a spherically symmetric tree $S$ which is such that $S_{n}$ is of the order $2^{n} n^{2}$. It follows from the above that $p_{\mathrm{c}}(S)=\frac{1}{2}$ and that $\theta_{S}\left(\frac{1}{2}\right)>0$. Next, we take 
a regular tree $S^{\prime}$ with common degree 4 . It is well known that $p_{\mathrm{c}}\left(S^{\prime}\right)=\frac{1}{3}$. We then construct a tree $S^{\prime \prime}$ by joining the roots of $S$ and $S^{\prime}$ by a single edge. It is easy to see that $p_{\mathrm{c}}\left(S^{\prime \prime}\right)=\frac{1}{3}$ and that $\theta_{S^{\prime \prime}}$ is discontinuous at $\frac{1}{2}$.

Theorem 1.1 along with Propositions 1.1 and 1.2 will be proved in Section 2. All the other results are proved in Section 3. The issues about continuity of the percolation function are discussed in Section 4.

\section{Proofs of Proposition 1.1, Theorem 1.1, and Proposition 1.2}

We start by defining the following useful probability generating function:

$$
h(n, s):=\sum_{j=0}^{\infty} \mathrm{P}\left(L_{n}=j\right) s^{j} \quad \text { for all } n \geq 1 .
$$

It is known (see [1]) that if $h^{\prime \prime}(n, 1)<\infty$ for every $n$ then, for all $n \geq 1$, we have

$$
\left(\mathrm{E}\left[T_{n}\right]^{-1}+\sum_{j=1}^{n} \frac{h^{\prime \prime}(j, 1)}{h^{\prime}(j, 1)} \mathrm{E}\left[T_{j}\right]^{-1}\right)^{-1} \leq \mathrm{P}\left(T_{n}>0\right) .
$$

Of course, we have

$$
h^{\prime}(n, 1)=\sum_{j=0}^{\infty} j \mathrm{P}\left(L_{n}=j\right)=\mathrm{E}\left[L_{n}\right]
$$

and

$$
h^{\prime \prime}(n, 1)=\sum_{j=0}^{\infty} j(j-1) \mathrm{P}\left(L_{n}=j\right)=\mathrm{E}\left[L_{n}^{2}\right]-\mathrm{E}\left[L_{n}\right] .
$$

We can now proceed with the proof of Proposition 1.1.

Proof of Proposition 1.1. The proof of the first statement is easy. Assume that

$$
\liminf _{n \rightarrow \infty}\left(\mathrm{E}\left[T_{n}\right]\right)^{1 / n}=a<1 .
$$

Then we find that, for any $\varepsilon>0$ such that $a(1+\varepsilon)<1$, there exists a sequence $\left\{n_{k}\right\}_{k=1}^{\infty}$ such that

$$
\mathrm{P}\left(T_{n_{k}}>0\right) \leq \mathrm{E}\left[T_{n_{k}}\right] \leq(a(1+\varepsilon))^{n_{k}},
$$

so that

$$
\lim _{n \rightarrow \infty} \mathrm{P}\left(T_{n}>0\right)=0 .
$$

For the second statement, we start by observing that condition (1.1) gives $h^{\prime \prime}(n, 1)=\mathrm{E}\left[L_{n}^{2}\right]-$ $\mathrm{E}\left[L_{n}\right]<\infty$ for every $n$. Of course, this does not require the full statement of (1.1), which will be needed later. In turn, this shows us that inequality (2.1) is valid for every $n$ and, therefore, we need to prove that

$$
\begin{aligned}
& \limsup _{n \rightarrow \infty}\left(\mathrm{E}\left[T_{n}\right]^{-1}+\sum_{j=1}^{n} \frac{h^{\prime \prime}(j, 1)}{h^{\prime}(j, 1)} \mathrm{E}\left[T_{j}\right]^{-1}\right)^{-1} \\
& \quad=\limsup _{n \rightarrow \infty}\left(\mathrm{E}\left[T_{n}\right]^{-1}+\sum_{j=1}^{n} \frac{\mathrm{E}\left[L_{j}^{2}\right]-\mathrm{E}\left[L_{j}\right]}{\mathrm{E}\left[L_{j}\right]} \mathrm{E}\left[T_{j}\right]^{-1}\right)^{-1} \\
& \quad>0
\end{aligned}
$$


To this end, we observe that, by (1.1) and (1.2),

$$
\sup _{j} \frac{\mathrm{E}\left[L_{j}^{2}\right]-\mathrm{E}\left[L_{j}\right]}{\mathrm{E}\left[L_{j}\right]} \leq \frac{C_{1}}{C_{2}}=C<\infty .
$$

Since $\liminf _{n \rightarrow \infty}\left(\mathrm{E}\left[T_{n}\right]\right)^{1 / n}>1$, there exists a constant $b>1$ and an $N$ such that, for all $n \geq N$,

$$
\mathrm{E}\left[T_{n}\right]>b^{n}
$$

Therefore, for some constant $D<\infty$,

$$
\begin{aligned}
\mathrm{E}\left[T_{n}\right]^{-1}+\sum_{j=1}^{n} \frac{h^{\prime \prime}(j, 1)}{h^{\prime}(j, 1)} \mathrm{E}\left[T_{j}\right]^{-1} & \leq \mathrm{E}\left[T_{n}\right]^{-1}+C \sum_{j=1}^{n} \mathrm{E}\left[T_{j}\right]^{-1} \\
& \leq D+C \sum_{j=N}^{\infty} b^{-j} \\
& <\infty
\end{aligned}
$$

Since the right-hand side of the above inequality is independent of $n$, inequality (2.2) is valid. This completes the proof.

We continue by proving Proposition 1.2.

Proof of Proposition 1.2. Let $\left\{X_{i}\right\}_{i \geq 1}$ be i.i.d. with distribution according to $T_{n}$, conditioned on the event that $T_{\ell}=1$. Observe that, for $n \geq \ell$,

$$
T_{n}=\sum_{k=1}^{T_{\ell}} X_{k}
$$

so that (using Wald's lemma)

$$
\mathrm{E}\left[X_{1}\right]=\mathrm{E}\left[T_{n} \mid T_{\ell}=1\right]=\frac{\mathrm{E}\left[T_{n}\right]}{\mathrm{E}\left[T_{\ell}\right]} .
$$

Observe that by (1.1) we can use inequality (2.1) to conclude that, for $n \geq \ell$,

$$
\left(\mathrm{E}\left[T_{n} \mid T_{\ell}=1\right]^{-1}+\sum_{j=\ell+1}^{n} \frac{h^{\prime \prime}(j, 1)}{h^{\prime}(j, 1)} \mathrm{E}\left[T_{j} \mid T_{\ell}=1\right]^{-1}\right)^{-1} \leq \mathrm{P}\left(T_{n}>0 \mid T_{\ell}=1\right) .
$$

We will show that there exists a sequence $\left\{n_{k}\right\}_{k=1}^{\infty}$ of increasing integers and a constant $C<\infty$ such that, for all $k \geq 1$ and all $n \geq n_{k}$,

$$
\mathrm{E}\left[T_{n} \mid T_{n_{k}}=1\right]^{-1}+\sum_{j=n_{k}+1}^{n} \frac{h^{\prime \prime}(j, 1)}{h^{\prime}(j, 1)} \mathrm{E}\left[T_{j} \mid T_{n_{k}}=1\right]^{-1} \leq C .
$$

This will give us, for all $k \geq 1$,

$$
\lim _{n \rightarrow \infty} \mathrm{P}\left(T_{n}>0 \mid T_{n_{k}}=1\right) \geq \frac{1}{C}
$$


proving the lemma. To that end, observe that, as in the proof of Lemma 1.1, there exists a constant $C_{3}$ such that, for $n \geq \ell$,

$$
\begin{aligned}
\mathrm{E}\left[T_{n} \mid T_{\ell}=1\right]^{-1}+\sum_{j=\ell+1}^{n} \frac{h^{\prime \prime}(j, 1)}{h^{\prime}(j, 1)} \mathrm{E}\left[T_{j} \mid T_{\ell}=1\right]^{-1} \\
\quad \leq \mathrm{E}\left[T_{n} \mid T_{\ell}=1\right]^{-1}+C_{3} \sum_{j=\ell+1}^{n} \mathrm{E}\left[T_{j} \mid T_{\ell}=1\right]^{-1} \\
\quad \leq\left(C_{3}+1\right) \sum_{j=\ell+1}^{n} \mathrm{E}\left[T_{j} \mid T_{\ell}=1\right]^{-1} \\
\quad=\left(C_{3}+1\right) \mathrm{E}\left[T_{\ell}\right] \sum_{j=\ell+1}^{n} \frac{1}{\mathrm{E}\left[T_{j}\right]}
\end{aligned}
$$

where we have used (2.3) in the last equality. Therefore, showing that there exists a sequence $\left\{n_{k}\right\}_{k=1}^{\infty}$ of increasing integers and a constant $C<\infty$ such that, for all $k$, we have

$$
\mathrm{E}\left[T_{n_{k}}\right] \sum_{j=n_{k}+1}^{\infty} \frac{1}{\mathrm{E}\left[T_{j}\right]} \leq C,
$$

will give us (2.5).

We divide the proof into three cases. First, however, define

$$
m:=\liminf _{n \rightarrow \infty} \mathrm{E}\left[T_{n}\right]^{1 / n}>1 .
$$

In the first case we have $\mathrm{E}\left[T_{n}\right]^{1 / n}<m$ for infinitely many $n$. We can then conclude that there exists an $n_{1}$, defined to be the largest integer such that $\mathrm{E}\left[T_{n_{1}}\right]^{1 / n_{1}}=\min _{n \geq 1} \mathrm{E}\left[T_{n}\right]^{1 / n}$. Having defined $n_{k}$, we can then define $n_{k+1}$ to be the largest integer greater than $n_{k}$ such that $\mathrm{E}\left[T_{n_{k+1}}\right]^{1 / n_{k+1}}=\min _{n>n_{k}} \mathrm{E}\left[T_{n}\right]^{1 / n}$. Let $\varepsilon_{k}$ be defined through $\mathrm{E}\left[T_{n_{k}}\right]^{1 / n_{k}}=m\left(1-\varepsilon_{k}\right)$. Observe that, by definition of $n_{k}, \mathrm{E}\left[T_{n}\right]^{1 / n} \geq m\left(1-\varepsilon_{k}\right)$ for every $n \geq n_{k}$ and also that $\varepsilon_{k}>0$ for every $k$, and finally that $\varepsilon_{k} \rightarrow 0$ as $k \rightarrow \infty$. Therefore,

$$
\begin{aligned}
\mathrm{E}\left[T_{n_{k}}\right] \sum_{j=n_{k}+1}^{\infty} \frac{1}{\mathrm{E}\left[T_{j}\right]} & \leq \mathrm{E}\left[T_{n_{k}}\right] \sum_{j=n_{k}+1}^{\infty} \frac{1}{\left(m\left(1-\varepsilon_{k}\right)\right)^{j}} \\
& =\left(m\left(1-\varepsilon_{k}\right)\right)^{n_{k}} \sum_{j=1}^{\infty} \frac{1}{\left(m\left(1-\varepsilon_{k}\right)\right)^{n_{k}+j}} \\
& =\sum_{j=1}^{\infty} \frac{1}{\left(m\left(1-\varepsilon_{k}\right)\right)^{j}} .
\end{aligned}
$$

There exists a $K$ such that $m\left(1-\varepsilon_{k}\right)>1$ for every $k \geq K$. For $k \geq K$, the right-hand side of the above equation is then bounded by some constant $D_{k}<\infty$. Furthermore, we can take $D_{k} \geq D_{k+1}$ and conclude that, for all $k \geq K$,

$$
\mathrm{E}\left[T_{n_{k}}\right] \sum_{j=n_{k}+1}^{\infty} \frac{1}{\mathrm{E}\left[T_{j}\right]} \leq D_{K}<\infty .
$$


For the second and third cases, we have $\mathrm{E}\left[T_{n}\right]^{1 / n}<m$ for only finitely many $n$. We can therefore find large enough $N$ so that $\mathrm{E}\left[T_{n}\right]^{1 / n} \geq m$ for every $n \geq N$. We have, for every $n$, $\mathrm{E}\left[T_{n}\right]^{1 / n}=m(1+a(n))$, where the sequence of numbers $\{a(n)\}_{n=1}^{\infty}$ is such that $a(n) \geq 0$ for every $n \geq N$.

The second case occurs if $\liminf _{n \rightarrow \infty}(1+a(n))^{n}=C_{4}$ for some constant $C_{4}<\infty$. Then there exists a sequence of strictly increasing integers $\left\{n_{k}\right\}_{k=1}^{\infty}$ such that $\left(1+a\left(n_{k}\right)\right)^{n_{k}} \leq 2 C_{4}$ for every $k \geq 1$. By also requiring that $n_{1} \geq N$ we obtain

$$
\begin{aligned}
\mathrm{E}\left[T_{n_{k}}\right] \sum_{j=n_{k}+1}^{\infty} \frac{1}{\mathrm{E}\left[T_{j}\right]} & \leq \mathrm{E}\left[T_{n_{k}}\right] \sum_{j=n_{k}+1}^{\infty} \frac{1}{m^{j}} \\
& =m^{n_{k}}\left(1+a\left(n_{k}\right)\right)^{n_{k}} \sum_{j=1}^{\infty} \frac{1}{m^{n_{k}+j}} \\
& \leq 2 C_{4} \sum_{j=1}^{\infty} \frac{1}{m^{j}} \\
& <\infty .
\end{aligned}
$$

The third case occurs if $\lim _{n \rightarrow \infty}(1+a(n))^{n}=\infty$. We can then find a sequence $\left\{n_{k}\right\}_{k=1}^{\infty}$ (much as in the first case) such that, for every $k \geq 1,(1+a(n))^{n} \geq\left(1+a\left(n_{k}\right)\right)^{n_{k}}$ for every $n \geq n_{k}$. By again requiring that $n_{1} \geq N$ we obtain

$$
\begin{aligned}
\mathrm{E}\left[T_{n_{k}}\right] \sum_{j=n_{k}+1}^{\infty} \frac{1}{\mathrm{E}\left[T_{j}\right]} & \leq \mathrm{E}\left[T_{n_{k}}\right] \sum_{j=n_{k}+1}^{\infty} \frac{1}{\left(m\left(1+a\left(n_{k}\right)\right)\right)^{j}} \\
& =\frac{\left(m\left(1+a\left(n_{k}\right)\right)\right)^{n_{k}}}{\left(m\left(1+a\left(n_{k}\right)\right)\right)^{n_{k}}} \sum_{j=1}^{\infty} \frac{1}{\left(m\left(1+a\left(n_{k}\right)\right)\right)^{j}} \\
& \leq \sum_{j=1}^{\infty} \frac{1}{m^{j}} \\
& <\infty .
\end{aligned}
$$

We can therefore conclude that there exists a constant $C=C\left(\left\{L_{n}\right\}_{n=1}^{\infty}\right)<\infty$ and a sequence of strictly increasing integers $\left\{n_{k}\right\}_{k=1}^{\infty}$ such that, for all $k \geq 1$,

$$
\mathrm{E}\left[T_{n_{k}}\right] \sum_{j=n_{k}+1}^{\infty} \frac{1}{\mathrm{E}\left[T_{j}\right]} \leq C .
$$

This completes the proof.

We are now ready to prove Theorem 1.1.

Proof of Theorem 1.1. Using the fact that $p_{\mathrm{c}}(I)^{-1}=p_{\mathrm{c}}(\bar{T})^{-1}=\operatorname{br} \bar{T}$, we need to show that

$$
p_{\mathrm{c}}(I)^{-1}=\liminf _{n \rightarrow \infty}\left(\mathrm{E}\left[T_{n}\right]\right)^{1 / n} .
$$

We will do this by first proving that $p_{\mathrm{c}}(I)^{-1}=\liminf _{n \rightarrow \infty}\left(\theta_{n} \mathrm{E}\left[T_{n}\right]\right)^{1 / n}$ and then proving that

$$
\liminf _{n \rightarrow \infty}\left(\theta_{n} \mathrm{E}\left[T_{n}\right]\right)^{1 / n}=\liminf _{n \rightarrow \infty} \mathrm{E}\left[T_{n}\right]^{1 / n} .
$$


Consider the offspring distribution $L_{1}^{\prime}$ of the root of $I$. Let $T^{i}$ be the tree consisting of child number $i \in\left\{1, \ldots, L_{1}\right\}$ of the root of $T$ and all the descendants of this child. Also, define

$$
N_{1, \infty}=\left|\left\{T^{i}:\left|T^{i}\right|=\infty, i=1, \ldots, L_{1}\right\}\right| .
$$

It is not hard to see that, for $k \geq 1$,

$$
\mathrm{P}\left(L_{1}^{\prime}=k\right)=\mathrm{P}\left(N_{1, \infty}=k \mid N_{1, \infty} \geq 1\right)=\frac{\mathrm{P}\left(N_{1, \infty}=k\right)}{\theta} .
$$

Furthermore, letting $Y_{i}$ be i.i.d. Bin $\left(1, \theta_{1}\right)$ random variables and using Wald's lemma, we obtain

$$
\mathrm{E}\left[L_{1}^{\prime}\right]=\frac{1}{\theta} \mathrm{E}\left[N_{1, \infty}\right]=\frac{1}{\theta} \mathrm{E}\left[\sum_{i=1}^{L_{1}} Y_{i}\right]=\frac{1}{\theta} \mathrm{E}\left[Y_{1}\right] \mathrm{E}\left[L_{1}\right]=\frac{\theta_{1}}{\theta} \mathrm{E}\left[L_{1}\right] .
$$

Furthermore, this argument holds for any generation $n$ and, therefore, we have, for all $n \geq 1$,

$$
\mathrm{E}\left[L_{n}^{\prime}\right]=\frac{\theta_{n}}{\theta_{n-1}} \mathrm{E}\left[L_{n}\right]
$$

Now, perform independent percolation on $I$ with parameter $p$, thus creating a random graph that we denote by $\tau^{p}$. Recall that $I^{p}$ is the component of the root of this graph. Obviously, $I^{p}$ is the family tree of an inhomogeneous Galton-Watson process with some offspring distributions $\left\{L_{n}^{\prime \prime}\right\}_{n=1}^{\infty}$. Furthermore, trivially

$$
\mathrm{E}\left[L_{n}^{\prime \prime}\right]=p \mathrm{E}\left[L_{n}^{\prime}\right]=p \frac{\theta_{n}}{\theta_{n-1}} \mathrm{E}\left[L_{n}\right] \text { for all } n \geq 1 .
$$

Recall that $I_{n}^{p}$ is the number of vertices in $I^{p}$ at distance $n$ from the root, and recall that we defined $I_{n}$ similarly. We have, using a standard result from the theory of branching processes and (2.7),

$$
\mathrm{E}\left[I_{n}^{p}\right]=p^{n} \mathrm{E}\left[I_{n}\right]=p^{n} \prod_{i=1}^{n} \mathrm{E}\left[L_{i}^{\prime}\right]=p^{n} \prod_{i=1}^{n} \frac{\theta_{i}}{\theta_{i-1}} \mathrm{E}\left[L_{i}\right]=p^{n} \frac{\theta_{n}}{\theta} \mathrm{E}\left[T_{n}\right] .
$$

Therefore,

$$
\liminf _{n \rightarrow \infty}\left(\mathrm{E}\left[I_{n}^{p}\right]\right)^{1 / n}=p \liminf _{n \rightarrow \infty}\left(\frac{\theta_{n}}{\theta} \mathrm{E}\left[T_{n}\right]\right)^{1 / n}=p \liminf _{n \rightarrow \infty}\left(\theta_{n} \mathrm{E}\left[T_{n}\right]\right)^{1 / n} .
$$

We would like to use Proposition 1.1 and Proposition 1.2 on $I^{p}$. However, before we can do this, we need to show that the offspring distributions $\left\{L_{n}^{\prime \prime}\right\}_{n=1}^{\infty}$ satisfy conditions (1.1) and (1.2). When we use Proposition 1.2, we will assume that (1.3) is satisfied; the details will become clear.

For some vertex $x$ in generation $n-1$, let $T_{x}^{i}$ be the tree consisting of child number $i \in$ $\left\{1, \ldots, L_{n}\right\}$ of $x$ and all the descendants of this child. Define

$$
N_{n, \infty}=\left|\left\{T_{x}^{i}:\left|T_{x}^{i}\right|=\infty, i=1, \ldots, L_{n}\right\}\right|,
$$


and observe that the distribution of this random variable is trivially independent of the specific choice of $x$ in generation $n-1$. Let $Y_{i}^{n}$ be i.i.d. $\operatorname{Bin}\left(1, \theta_{n}\right)$, and observe that

$$
\begin{aligned}
\mathrm{E}\left[\left(L_{n}^{\prime \prime}\right)^{2}\right] & \leq \mathrm{E}\left[\left(L_{n}^{\prime}\right)^{2}\right] \\
& =\sum_{j=1}^{\infty} j^{2} \mathrm{P}\left(L_{n}^{\prime}=j\right) \\
& =\sum_{j=1}^{\infty} j^{2} \mathrm{P}\left(N_{n, \infty}=j \mid N_{n, \infty} \geq 1\right) \\
& =\frac{\mathrm{E}\left[N_{n, \infty}^{2}\right]}{\theta_{n-1}} \\
& =\frac{\mathrm{E}\left[\mathrm{E}\left[\left(\sum_{i=1}^{L_{n}} Y_{i}^{n}\right)^{2} \mid L_{n}\right]\right]}{\theta_{n-1}} \\
& \leq \frac{\mathrm{E}\left[\mathrm{E}\left[L_{n} \sum_{i=1}^{L_{n}}\left(Y_{i}^{n}\right)^{2} \mid L_{n}\right]\right]}{\theta_{n-1}} \\
& =\frac{\mathrm{E}\left[L_{n} \sum_{i=1}^{L_{n}} \mathrm{E}\left[Y_{i}^{n} \mid L_{n}\right]\right]}{\theta_{n-1}} \\
& =\frac{\mathrm{E}\left[L_{n}^{2} \theta_{n}\right]}{\theta_{n-1}} .
\end{aligned}
$$

In the second inequality we use the fact that, for any real numbers $a_{1}, \ldots, a_{n}$, we have $\left(a_{1}+\right.$ $\left.\cdots+a_{n}\right)^{2} \leq n\left(a_{1}^{2}+\cdots+a_{n}^{2}\right)$. Obviously, we must also have

$$
\theta_{n-1} \geq \mathrm{P}\left(L_{n}>0\right) \theta_{n},
$$

and we can use Cauchy-Schwarz to see that

$$
\mathrm{E}\left[L_{n}\right]^{2}=\mathrm{E}\left[L_{n} \mathbf{1}_{\left\{L_{n}>0\right\}}\right]^{2} \leq \mathrm{P}\left(L_{n}>0\right) \mathrm{E}\left[L_{n}^{2}\right] .
$$

Therefore,

$$
\frac{\mathrm{E}\left[L_{n}^{2} \theta_{n}\right]}{\theta_{n-1}} \leq \frac{\mathrm{E}\left[L_{n}^{2}\right]}{\mathrm{P}\left(L_{n}>0\right)} \leq \mathrm{E}\left[L_{n}^{2}\right] \frac{\mathrm{E}\left[L_{n}^{2}\right]}{\mathrm{E}\left[L_{n}\right]^{2}} \leq \frac{C_{1}^{2}}{C_{2}^{2}}<\infty
$$

Furthermore,

$$
\inf _{n} \mathrm{E}\left[L_{n}^{\prime \prime}\right]=p \inf _{n} \mathrm{E}\left[L_{n}^{\prime}\right] \geq p
$$

since $\mathrm{E}\left[L_{n}^{\prime}\right] \geq 1$ for every $n$.

We can now proceed to use Proposition 1.1 with (2.8) to see that $I^{p}$ survives with positive probability if $p>\left(\liminf _{n \rightarrow \infty}\left(\theta_{n} \mathrm{E}\left[T_{n}\right]\right)^{1 / n}\right)^{-1}$, while it dies out almost surely if $p<$ $\left(\liminf _{n \rightarrow \infty}\left(\theta_{n} \mathrm{E}\left[T_{n}\right]\right)^{1 / n}\right)^{-1}$.

This is not quite enough for our purposes: it could be the case that, with positive probability, $I$ is such that $I^{p}$ almost surely dies out. Since we want to make a statement about almost all trees $I$, we argue that, in fact, if $p>1 / \liminf _{n \rightarrow \infty}\left(\theta_{n} \mathrm{E}\left[T_{n}\right]\right)^{1 / n}$ then $\mathcal{L}^{p}$ contains an infinite component with probability 1 , as our next argument shows. 
Assume that $\liminf _{n \rightarrow \infty}\left(\mathrm{E}\left[I_{n}^{p}\right]\right)^{1 / n}=p \liminf _{n \rightarrow \infty}\left(\theta_{n} \mathrm{E}\left[T_{n}\right]\right)^{1 / n}>1$. This is condition (1.3) for $I^{p}$. Construct the tree $I^{p}$ by letting $I_{1}^{p}$ have distribution equal to $L_{1}^{\prime \prime}$. Proceed by letting $I_{2}^{p}$ be the sum

$$
\sum_{i=1}^{I_{1}^{p}} L_{2, i}^{\prime \prime}
$$

where $\left\{L_{2, i}^{\prime \prime}\right\}_{i=1}^{\infty}$ are i.i.d. with distribution equal to $L_{2}^{\prime \prime}$, and let them also be independent of everything else. Continuing in this fashion, we have two possibilities. First, we may find that $I_{n}^{p}>0$ for every $n$. Second, we might instead find that, for some $n$, we have $I_{n}^{p}=0$. If this is the case, there exists some integer $n_{k_{1}}>n$ in the subsequence dictated by Proposition 1.2. However, since $I$ is infinite, we see that $\tau^{p}$ must contain a subtree (possibly consisting of only one vertex ) with the root being some vertex at level $n_{k_{1}}$. Construct this subtree in the same way as we constructed $I^{p}$ above. This subtree has some probability to survive which is, by Proposition 1.2, uniformly bounded away from 0 . It is also easy to see that the event of survival of this subtree is conditionally independent of the part of $\tau^{p}$ examined so far (up to generation $n$ ).

If again we find that this subtree is finite, we continue in the same way. Since all the subtrees that we pick have uniformly positive probability to survive by Proposition 1.2 and the survival of them are conditionally independent, we see that $\mathcal{I}^{p}$ must contain an infinite component with probability 1 . We therefore conclude that

$$
\mathrm{P}\left(\mathcal{L}^{p} \text { has an infinite component }\right)= \begin{cases}1, & p>1 / \liminf _{n \rightarrow \infty}\left(\theta_{n} \mathrm{E}\left[T_{n}\right]\right)^{1 / n}, \\ 0, & p<1 / \liminf _{n \rightarrow \infty}\left(\theta_{n} \mathrm{E}\left[T_{n}\right]\right)^{1 / n} .\end{cases}
$$

This is the same as saying that, for almost every $I$, we will, after performing percolation with parameter $p$ on $I$, almost surely obtain an infinite component if

$$
p>\frac{1}{\liminf _{n \rightarrow \infty}\left(\theta_{n} \mathrm{E}\left[T_{n}\right]\right)^{1 / n}},
$$

while we will almost surely only obtain finite components if

$$
p<\frac{1}{\liminf _{n \rightarrow \infty}\left(\theta_{n} \mathrm{E}\left[T_{n}\right]\right)^{1 / n}} .
$$

It follows that, for almost every $I$, the probability that the component of the root is infinite is positive if $p>1 / \liminf _{n \rightarrow \infty}\left(\theta_{n} \mathrm{E}\left[T_{n}\right]\right)^{1 / n}$, while it is 0 if $p<1 / \liminf _{n \rightarrow \infty}\left(\theta_{n} \mathrm{E}\left[T_{n}\right]\right)^{1 / n}$. This gives us

$$
p_{\mathrm{c}}(I)=\frac{1}{\liminf _{n \rightarrow \infty}\left(\theta_{n} \mathrm{E}\left[T_{n}\right]\right)^{1 / n}},
$$

from which it follows that $\operatorname{br} I=\liminf _{n \rightarrow \infty}\left(\theta_{n} \mathrm{E}\left[T_{n}\right]\right)^{1 / n}\left(\right.$ recall that $\left.p_{\mathrm{c}}(I)=1 / \operatorname{br} I\right)$.

We now proceed with the final step in proving that

$$
\liminf _{n \rightarrow \infty}\left(\theta_{n} \mathrm{E}\left[T_{n}\right]\right)^{1 / n}=\liminf _{n \rightarrow \infty} \mathrm{E}\left[T_{n}\right]^{1 / n} .
$$

Obviously, $\theta_{n} \mathrm{E}\left[T_{n}\right] \leq \mathrm{E}\left[T_{n}\right]$ for every $n$, so we only need to show that

$$
\liminf _{n \rightarrow \infty}\left(\theta_{n} \mathrm{E}\left[T_{n}\right]\right)^{1 / n} \geq \liminf _{n \rightarrow \infty} \mathrm{E}\left[T_{n}\right]^{1 / n} .
$$


As before, let $m=\liminf _{n \rightarrow \infty} \mathrm{E}\left[T_{n}\right]^{1 / n}>1$, and choose $\varepsilon>0$ so that $m(1-\varepsilon)>1$. Furthermore, we can choose an $N$ such that $\mathrm{E}\left[T_{n}\right]^{1 / n} \geq m(1-\varepsilon)$ for every $n \geq N$. Using inequalities (2.4) and (2.6), we obtain, for some constant $C$ and $m \geq n$,

$$
\mathrm{P}\left(T_{m}>0 \mid T_{n}=1\right) \geq\left(C \mathrm{E}\left[T_{n}\right] \sum_{j=n+1}^{m} \frac{1}{\mathrm{E}\left[T_{j}\right]}\right)^{-1} \geq\left(C \mathrm{E}\left[T_{n}\right] \sum_{j=n+1}^{\infty} \frac{1}{\mathrm{E}\left[T_{j}\right]}\right)^{-1} .
$$

Therefore, for $n \geq N$,

$$
\begin{aligned}
\theta_{n} \mathrm{E}\left[T_{n}\right] & =\lim _{m \rightarrow \infty} \mathrm{P}\left(T_{m}>0 \mid T_{n}=1\right) \mathrm{E}\left[T_{n}\right] \\
& \geq\left(C \sum_{j=n+1}^{\infty} \frac{1}{\mathrm{E}\left[T_{j}\right]}\right)^{-1} \\
& \geq\left(C \sum_{j=n+1}^{\infty} \frac{1}{(m(1-\varepsilon))^{j}}\right)^{-1} \\
& =\left(\frac{C}{(m(1-\varepsilon))^{n}} \sum_{j=1}^{\infty} \frac{1}{(m(1-\varepsilon))^{j}}\right)^{-1} \\
& =(m(1-\varepsilon))^{n} C^{\prime},
\end{aligned}
$$

where $C^{\prime}>0$. Therefore, for all $n \geq N$, we have

$$
\left(\theta_{n} \mathrm{E}\left[T_{n}\right]\right)^{1 / n} \geq m(1-\varepsilon) C^{1 / n},
$$

so that

$$
\liminf _{n \rightarrow \infty}\left(\theta_{n} \mathrm{E}\left[T_{n}\right]\right)^{1 / n} \geq m(1-\varepsilon) .
$$

Since $\varepsilon>0$ can be chosen arbitrarily small, this completes the proof.

Remark 2.1. In fact, the proof of Theorem 1.1 shows that if the family tree $T$ of a GaltonWatson process satisfies $(1.1),(1.2)$, and $(1.3)$, and $p>p_{\mathrm{c}}(\bar{T})$, then so does the family tree associated with the $I^{p}$ process.

\section{Proofs of Theorem 1.2 and Proposition 1.3}

Before we can prove Theorem 1.2, we need the following domination lemmas. The first one appears (without proof) in [2]. The proof we give is due to Olle Häggström (unpublished).

Lemma 3.1. For $k \geq 1, p \in(0,1)$, and $0 \leq m \leq k$, write $\rho_{k, p, m}$ for the distribution of $a$ $\operatorname{Bin}(k, p)$ random variable conditioned on taking a value of at least $m$. For $p_{1} \leq p_{2}$, we have

$$
\rho_{k, p_{1}, m} \preceq \rho_{k, p_{2}, m},
$$

where ' $\preceq$ 'denotes 'stochastic domination'.

Proof. For $i=1,2$, let $Y_{i}$ be a $\operatorname{Bin}\left(k, p_{i}\right)$ random variable, and let $X_{i}$ be a random variable with distribution $\rho_{k, p_{i}, m}$. Since $x /(1-x)<y /(1-y)$ for $0<x<y<1$, it is enough to show that, for any $n \in\{m+1, \ldots, k\}$, we have

$$
\frac{\mathrm{P}\left(X_{1} \geq n\right)}{\mathrm{P}\left(X_{1}<n\right)} \leq \frac{\mathrm{P}\left(X_{2} \geq n\right)}{\mathrm{P}\left(X_{2}<n\right)}
$$


which is the same as showing that

$$
\frac{\mathrm{P}\left(X_{2} \geq n\right)}{\mathrm{P}\left(X_{1} \geq n\right)} \frac{\mathrm{P}\left(X_{1}<n\right)}{\mathrm{P}\left(X_{2}<n\right)} \geq 1 .
$$

Writing $Z_{1}$ and $Z_{2}$ for the probabilities that $Y_{1} \geq m$ and $Y_{2} \geq m$, respectively, the left-hand side of (3.1) becomes

$$
\frac{\left(1 / Z_{2}\right) \sum_{j=n}^{k}\left(\begin{array}{c}
k \\
j
\end{array}\right) p_{2}^{j}\left(1-p_{2}\right)^{k-j}}{\left(1 / Z_{1}\right) \sum_{j=n}^{k}\left(\begin{array}{c}
k \\
j
\end{array}\right) p_{1}^{j}\left(1-p_{1}\right)^{k-j}} \frac{\left(1 / Z_{1}\right) \sum_{j=m}^{n-1}\left(\begin{array}{c}
k \\
j
\end{array}\right) p_{1}^{j}\left(1-p_{1}\right)^{k-j}}{\left(1 / Z_{2}\right) \sum_{j=m}^{n-1}\left(\begin{array}{c}
k \\
j
\end{array}\right) p_{2}^{j}\left(1-p_{2}\right)^{k-j}} .
$$

Cancelling the $Z_{i}$ s and introducing the notation $\phi_{i}=p_{i} /\left(1-p_{i}\right)$ for $i=1,2$, the expression in (3.2) may be further rewritten as

$$
\begin{aligned}
& \frac{p_{2}^{n}\left(1-p_{2}\right)^{k-n} \sum_{j=n}^{k}\left(\begin{array}{c}
k \\
j
\end{array}\right) \phi_{2}^{j-n}}{p_{1}^{n}\left(1-p_{1}\right)^{k-n} \sum_{j=n}^{k}\left(\begin{array}{c}
k \\
j
\end{array}\right) \phi_{1}^{j-n}} \frac{p_{1}^{n}\left(1-p_{1}\right)^{k-n} \sum_{j=m}^{n-1}\left(\begin{array}{c}
k \\
j
\end{array}\right) \phi_{1}^{j-n}}{p_{2}^{n}\left(1-p_{2}\right)^{k-n} \sum_{j=m}^{n-1}\left(\begin{array}{c}
k \\
j
\end{array}\right) \phi_{2}^{j-n}} \\
& =\frac{\sum_{j=n}^{k}\left(\begin{array}{l}
k \\
j
\end{array}\right) \phi_{2}^{j-n}}{\sum_{j=n}^{k}\left(\begin{array}{c}
k \\
j
\end{array}\right) \phi_{1}^{j-n}} \frac{\sum_{j=m}^{n-1}\left(\begin{array}{c}
k \\
j
\end{array}\right) \phi_{1}^{j-n}}{\sum_{j=m}^{n-1}\left(\begin{array}{c}
k \\
j
\end{array}\right) \phi_{2}^{j-n}} \text {. }
\end{aligned}
$$

Now note that $\phi_{1} \leq \phi_{2}$, so that

$$
\sum_{j=n}^{k}\left(\begin{array}{l}
k \\
j
\end{array}\right) \phi_{2}^{j-n} \geq \sum_{j=n}^{k}\left(\begin{array}{l}
k \\
j
\end{array}\right) \phi_{1}^{j-n}
$$

and

$$
\sum_{j=m}^{n-1}\left(\begin{array}{l}
k \\
j
\end{array}\right) \phi_{1}^{j-n} \geq \sum_{j=m}^{n-1}\left(\begin{array}{l}
k \\
j
\end{array}\right) \phi_{2}^{j-n}
$$

Hence, the expression in (3.3) is greater than or equal to 1 , so (3.1) is verified and the lemma is established.

We proceed with the following lemma. We will in fact only use it for the case in which $m=1$, but we nevertheless provide a proof of the general statement.

Lemma 3.2. In the notation of Lemma 3.1, it is the case that, for any $1 \leq k \leq l$ and $0 \leq m \leq k$,

$$
\rho_{k, p, m} \preceq \rho_{l, p, m} \text { for all } 0<p<1 .
$$

Proof. It is obvious that we only need to prove the lemma for the case in which $l=$ $k+1$. Therefore, let $Y_{1}, \ldots, Y_{k+1}$ and $X_{1}, \ldots, X_{k}$ be i.i.d. Bernoulli random variables with expectation $p$, and let $Y=\sum_{i=1}^{k+1} Y_{i}$ and $X=\sum_{j=1}^{k} X_{j}$. We need to show that $\mathrm{P}(X \geq n \mid X \geq$ $m) \leq \mathrm{P}(Y \geq n \mid Y \geq m)$ for all $n=m, m+1, \ldots, k$. To this end, we write

$$
\begin{aligned}
\mathrm{P}(Y \geq n \mid Y \geq m)= & \mathrm{P}\left(Y \geq n \mid Y \geq m, Y_{k+1}=0\right) \mathrm{P}\left(Y_{k+1}=0 \mid Y \geq m\right) \\
& +\mathrm{P}\left(Y \geq n \mid Y \geq m, Y_{k+1}=1\right) \mathrm{P}\left(Y_{k+1}=1 \mid Y \geq m\right) \\
= & \mathrm{P}(X \geq n \mid X \geq m) \mathrm{P}\left(Y_{k+1}=0 \mid Y \geq m\right) \\
& +\mathrm{P}(X \geq n-1 \mid X \geq m-1) \mathrm{P}\left(Y_{k+1}=1 \mid Y \geq m\right) .
\end{aligned}
$$


Therefore, we need to show that, for $n>m$,

$$
\mathrm{P}(X \geq n-1 \mid X \geq m-1) \geq \mathrm{P}(X \geq n \mid X \geq m),
$$

or, equivalently,

$$
\mathrm{P}(X \geq n \mid X \geq n-1) \leq \mathrm{P}(X \geq m \mid X \geq m-1) .
$$

It is easy to see that it suffices to prove this for $m=n-1$, or to simplify the notation, to show that

$$
\mathrm{P}(X \geq n+1 \mid X \geq n) \leq \mathrm{P}(X \geq n \mid X \geq n-1) .
$$

Since

$$
\mathrm{P}(X \geq n+1 \mid X \geq n)=1-\mathrm{P}(X=n \mid X \geq n),
$$

we need to show that

$$
\mathrm{P}(X=n-1 \mid X \geq n-1) \leq \mathrm{P}(X=n \mid X \geq n) .
$$

Writing $p_{n}:=\mathrm{P}(X=n)$, we rewrite this as

$$
\frac{p_{n}+\cdots+p_{k}}{p_{n-1}+\cdots+p_{k}} \leq \frac{p_{n}}{p_{n-1}},
$$

or, equivalently, as

$$
p_{n-1}\left(p_{n+1}+\cdots+p_{k}\right) \leq p_{n}\left(p_{n}+\cdots+p_{k}\right) .
$$

It suffices to show that $p_{n-1} p_{n+j} \leq p_{n} p_{n+j-1}$ for $1 \leq j \leq k-n$. This, however, is easily checked by a straightforward calculation.

We are now ready to prove Theorem 1.2.

Proof of Theorem 1.2. For the purpose of this proof, we introduce a new stochastic process $\tilde{I}_{n}^{p}$, indexed by $n=1,2, \ldots$, as follows. Let $\tilde{I}_{1}^{p}$ be distributed as the number of points in $I_{1}^{p}$. However, if this number of points is 0 , we resample according to the same distribution and repeat this until the total number of offspring is at least equal to 1 . If we do not resample at this first generation, we define $R_{0}:=1$; if we do resample, we set $R_{0}=0$.

In an inductive fashion, having defined $\tilde{I}_{n}^{p}$, we consider all points in $\tilde{I}_{n}^{p}$ and give each of them a random number of offspring distributed as $L_{n+1}^{\prime \prime}$, independently of each other. However, if the total number of offspring is 0 , we resample all offspring using the same distributions, until the total number of offspring is at least equal to 1 . If we do not have to resample, we define $R_{n}:=1$; if we do resample, we set $R_{n}=0$. Of course, the distribution of the number of points in $\tilde{I}_{n}^{p}$ given $\tilde{I}_{n-1}^{p}=k$ for some $k \geq 1$ is the same as the distribution of the number of points in $I_{n}^{p}$ given $I_{n-1}^{p}=k$ conditioned on being at least equal to 1 .

We can now write, for any $M$,

$$
\begin{aligned}
\mathrm{P}\left(0<I_{n}^{p}<M\right) & =\mathrm{P}\left(\prod_{i=0}^{n-1} R_{i}=1,0<\tilde{I}_{n}^{p}<M\right) \\
& \leq \mathrm{P}\left(0<\tilde{I}_{n}^{p}<M\right) \\
& =\mathrm{P}\left(\tilde{I}_{n}^{p}<M\right) .
\end{aligned}
$$


Now let $p_{\mathrm{c}}(\bar{T})<p<q$. We claim that

$$
\tilde{I}_{n}^{p} \preceq \tilde{I}_{n}^{q} .
$$

To see this, we note that the offspring distributions of $I^{p}$ can be realized by first drawing from the appropriate $L_{n}^{\prime}$ and then keeping all points in the offspring with probability $p$, independently of each other. Now the combination of Lemma 3.1 and Lemma 3.2 implies that, for $k \leq \ell$ and $p \leq q$, we have

$$
\rho_{k, p, 1} \preceq \rho_{\ell, q, 1} .
$$

Clearly, we can couple $\tilde{I}_{1}^{p}$ and $\tilde{I}_{1}^{q}$ so that $\tilde{I}_{1}^{p} \leq \tilde{I}_{1}^{q}$, since we can use the same offspring $L_{1}^{\prime}$ for them to obtain $I_{1}$ and then the domination follows from Lemma 3.1. Let $\left\{L_{2, i}^{\prime}\right\}_{i=1}^{\tilde{I}_{1}^{q}}$ be i.i.d. with distribution equal to $L_{2}^{\prime}$ and independent of everything else. We can now obtain $\tilde{I}_{2}^{p}$ by letting it be a $\operatorname{Bin}\left(\sum_{i=1}^{\tilde{I}_{1}^{p}} L_{2, i}^{\prime}, p\right)$ conditioned on being at least equal to 1 . Similarly, we obtain $\tilde{I}_{2}^{q}$ by letting it be a $\operatorname{Bin}\left(\sum_{i=1}^{\tilde{I}_{1}^{q}} L_{2, i}^{\prime}, q\right)$ conditioned on being at least equal to 1 . The fact that we can couple $\tilde{I}_{2}^{q}$ and $\tilde{I}_{2}^{p}$ so that $\tilde{I}_{2}^{q} \leq \tilde{I}_{2}^{p}$ now follows from (3.5). Repeating this procedure at every level gives

$$
\mathrm{P}\left(\tilde{I}_{n}^{p}<M\right) \leq \mathrm{P}\left(\tilde{I}_{n}^{p_{1}}<M\right) \text { for all } p>p_{1},
$$

and this is where the uniformity in $p$ comes from.

Of course, letting $M$ above depend on $n$ does not change the validity of the argument. According to (3.4) and (3.6) it therefore suffices to show that

$$
\mathrm{P}\left(\tilde{I}_{n}^{p_{1}}<\left((1-\varepsilon) \operatorname{br} I^{p_{1}}\right)^{n}\right) \rightarrow 0 \quad \text { as } n \rightarrow \infty .
$$

For this, we use Theorem 1.1 and Proposition 1.2. Consider the subsequence $\left\{n_{k}\right\}$ and the constant $C>0$ dictated by applying Proposition 1.2 to $I^{p_{1}}$. This is allowed according to Remark 2.1. Since each element in the $n_{1}$ th generation of the $I^{p_{1}}$ process has a probability of at least $C$ to survive, there is at least probability $C>0$ that no resampling is ever going to be necessary in the $\tilde{I}^{p_{1}}$ process after time $n_{1}$. There are now two possibilities. Either at some point resampling is needed or no resampling is ever needed after time $n_{1}$.

In the latter case, $\tilde{I}_{n}^{p_{1}}$ is at least as large as the number of points in a surviving copy of an $I^{p_{1}}$ tree with only one vertex at generation $n_{1}$. It follows from Theorem 1.1 that this surviving tree has branching number br $I^{p_{1}}$. Using the fact that the lower growth number is at least as large as the branching number, we are done in this case.

On the other hand, if resampling is needed then we take the first element in the subsequence $\left\{n_{k}\right\}$ after the first resampling and repeat the reasoning from there. It follows that, almost surely, $\liminf _{n \rightarrow \infty}\left(\tilde{I}_{n}^{p_{1}}\right)^{1 / n} \geq \operatorname{br} \bar{I}^{\bar{p}_{1}}$, and the proof is complete.

We can now prove Proposition 1.3.

Proof of Proposition 1.3. We write

$$
\theta(p)=\mathrm{P}\left(I_{n}^{p}>0\right)-\mathrm{P}\left(I_{n}^{p}>0,\left|I^{p}\right|<\infty\right)
$$

(recall that $I^{p}$ denotes the component of the root). We will prove that, along a subsequence, the last term tends to 0 uniformly in $p \in\left[p_{1}, 1\right]$, where $p_{1}>p_{\mathrm{c}}(\bar{T})$, from which the result follows. 
Since the $p$-dependence is important now, we write $\theta_{n}(p)$ for $\theta_{n}$ in the context of the GaltonWatson process associated with $I^{p}$. For any $M>0$, we write, for $p_{1} \leq p \leq 1$,

$$
\begin{aligned}
\mathrm{P}\left(I_{n}^{p}>0,\left|I^{p}\right|<\infty\right) & \leq \mathrm{P}\left(0<I_{n}^{p}<M\right)+\mathrm{P}\left(I_{n}^{p} \geq M,\left|I^{p}\right|<\infty\right) \\
& \leq \mathrm{P}\left(0<I_{n}^{p}<M\right)+\left(1-\theta_{n}(p)\right)^{M} \\
& \leq \mathrm{P}\left(0<I_{n}^{p}<M\right)+\left(1-\theta_{n}\left(p_{1}\right)\right)^{M} .
\end{aligned}
$$

Let $\varepsilon>0$ be arbitrary. We want to apply Proposition 1.2 to $I^{p}$. According to Remark 2.1, all the assumptions of Proposition 1.2 hold for $I^{p}$ since $p>p_{\mathrm{c}}(\bar{T})$.

Now let $C$ be the constant in Proposition 1.2 when we apply it to $I^{p_{1}}$. We choose $M$ so large that $(1-C)^{M}<\varepsilon / 2$. Next choose $n$ in the appropriate subsequence of Proposition 1.2 and at the same time choose it to be so large that the first term on the right-hand side of the above inequality is at most $\varepsilon / 2$; this is possible according to Theorem 1.2. The right-hand side is then bounded above by $\varepsilon$, uniformly in $p \in\left[p_{1}, 1\right]$. In summary, for any $\varepsilon>0$, we can find a $K$ such that

$$
\theta(p) \geq \mathrm{P}\left(I_{n_{k}}^{p}>0\right)-\varepsilon
$$

for every $p \in\left[p_{1}, 1\right]$ and every $n_{k}$ in the subsequence dictated by Proposition 1.2 with $k \geq K$. We see that, for all $k \geq K$ and all $p \in\left[p_{1}, 1\right]$,

$$
\left|\theta(p)-\mathrm{P}\left(I_{n_{k}}^{p}>0\right)\right| \leq \varepsilon .
$$

This completes the proof.

\section{Continuity of the percolation function}

The supercritical continuity of $\theta(p)$ (Corollary 1.1) follows immediately from Proposition 1.3. We point out, however, that it is possible to obtain the same result by combining Theorem 1.1 with a modified version of the classical argument found in [7]. We provide a sketch.

Sketch of proof of Corollary 1.1 from Theorem 1.1. We start by drawing an I from the correct distribution. Associate to every edge $e$ in $I$ an independent $U([0,1])$ random variable, denoted by $U_{e}$. For $p_{\mathrm{c}}<q<p$, create $\mathcal{I}^{q}$ and $\mathcal{I}^{p}$ by keeping every vertex of $I$ and those edges $e \in I$ such that $U_{e} \leq q, p$, respectively. Consider any infinite subtree $J$ in $\tau^{p}$. Theorem 1.1 gives $p_{\mathrm{c}}(J)=1 / \mathrm{br} J=1 /\left(p \liminf _{n \rightarrow \infty} \mathrm{E}\left[I_{n}\right]^{1 / n}\right)=p_{\mathrm{c}}(I) / p$ almost surely. Therefore, performing further percolation on $J$ with density $q / p>p_{\mathrm{c}}(I) / p$ will result in a new graph containing an infinite subgraph almost surely. Of course, the distribution of this new graph must be the same as $J \cap \mathcal{I}^{q}$. Furthermore, this holds in particular if $J=I^{p}$, showing that if $\left|I^{p}\right|=\infty$ then there almost surely exists an infinite subtree of $I^{p} \cap I^{q}$. It is now possible to proceed as in [7].

The nonclassical way to conclude continuity of the percolation function has an interesting analogy on $\mathbb{Z}^{d}$. Define $B_{n}:=[-n, n]^{d}$, and write $\partial B_{n}$ for the (inner) boundary of $B_{n}$. Letting $\left\{0 \leftrightarrow \partial B_{n}\right\}$ denote the event that the origin is connected to $\partial B_{n}$ by a path of open edges, define

$$
\varphi_{n}(p):=\mathrm{P}_{p}\left(0 \leftrightarrow \partial B_{n}\right) .
$$

Clearly,

$$
\theta(p)=\lim _{n \rightarrow \infty} \varphi_{n}(p) \quad \text { for all } 0 \leq p \leq 1
$$


The inequality of the following equation (valid for every $n \geq 1$ ) is a part of Theorem 8.18 of [3]:

$$
\varphi_{n}(p)-\theta(p)=\mathrm{P}_{p}\left(0 \leftrightarrow B_{n},|C|<\infty\right) \leq A(p, d) n^{d} \mathrm{e}^{-n \sigma(p)},
$$

where we can take

$$
A(p, d)=\frac{d^{2}}{p^{2}(1-p)^{d-2}} .
$$

Furthermore, according to Theorem 8.21 of [3] we can take $\sigma(p)$ to be uniformly bounded away from 0 on any closed subinterval of $\left(p_{\mathrm{c}}, 1\right)$. We point out the following corollary and sketch its proof.

Corollary 4.1. The percolation function $\theta(p)$ on $\mathbb{Z}^{d}, d \geq 2$, is continuous for $p>p_{\mathrm{c}}$.

Sketch of proof. Choose $p_{\mathrm{c}}<p_{1}<p_{2}<1$. Combining (4.1), (4.2), and Theorem 8.21 of [3] explained directly above, it is straightforward to prove that there exist constants $C=$ $C\left(p_{1}, p_{2}\right)<\infty$ and $\delta=\delta\left(p_{1}, p_{2}\right)>0$ such that, for any $p \in\left[p_{1}, p_{2}\right]$ and any $n \geq 1$,

$$
\varphi_{n}(p)-\theta(p) \leq C \mathrm{e}^{-n \delta} .
$$

Since, trivially,

$$
\theta(p) \leq \varphi_{n}(p),
$$

it follows that $\varphi_{n}(p) \rightarrow \theta(p)$ uniformly on any closed subinterval of $\left(p_{\mathrm{c}}, 1\right)$, from which the statement follows.

\section{References}

[1] Agresti, A. (1975). On the extinction times of varying and random environment branching processes. J. Appl. Prob. 12, 39-46.

[2] Broman, E. I., Häggström, O. and Steif, J. E. (2006). Refinements of stochastic domination. Prob. Theory Relat. Fields 136, 587-603.

[3] Grimmett, G. (1999). Percolation, 2nd edn. Springer, Berlin.

[4] Lyons, R. (1990). Random walks and percolation on trees. Ann. Prob. 18, 931-958.

[5] Lyons, R. (1992). Random walks, capacity and percolation on trees. Ann. Prob. 20, 2043-2088.

[6] LyOns, R. Probability on trees and networks. In preparation. Available at http://mypage.iu.edu/ rdlyons/ prbtree/prbtree.html.

[7] VAN DEN Berg, J. AND KeAne, M. (1982). On the continuity of the percolation probability function. In Conference in Modern Analysis and Probability, eds R. Beats et al., AMS, Providence, RI, pp. 61-65. 